\title{
Synthesis of polyhydroquinoline derivatives under aqueous media
}

\author{
B. P. Bandgar,* P. E. More, V. T. Kamble, and J. V. Totre \\ Organic Chemistry Research Laboratory, School of Chemical Sciences, \\ Swami Ramanand Teerth Marathwada University, Vishnupuri, Nanded-431 606, India Email: \\ bandgar_bp@yahoo.com
}

\begin{abstract}
Highly efficient synthesis of polyhydroquinoline derivatives was reported via four component reaction of aldehydes, dimedone, ethyl acetoacetate, and ammonium acetate in refluxing water. Excellent yields of the products and simple work-up are attractive features of this green protocol.
\end{abstract}

Keywords: Dimedone, aldehydes, polyhydroquinoline, multi-component reactions, aqueous media

\section{Introduction}

The environmental acceptability of the process is improved if the multi-component strategy is applied. Indeed, it is well known that multi-component reactions (MCR), consisting of two or more synthetic steps, which are carried out without isolation of any intermediate, allow to reduce time, save money, energy and raw materials. ${ }^{1}$ In addition, aqueous mediated reactions have received considerable attention in organic synthesis due to environmental safety reasons. Water is the most abundant, cheapest, and non-toxic chemical in nature. It has high dielectric constant and cohesive energy density compared to organic solvents. It has also special effects on reactions arising from inter and intramolecular non-covalent interactions leading to novel solvation and assembly processes. Water as a reaction medium have been utilized for large numbers of organic reactions. ${ }^{2}$ Thus, development of an efficient and convenient synthetic methodology in aqueous medium is an important area of research.

In recent years, much attention has been focused on the synthesis of 1,4-dihydropyridyl compounds because of their variety of pharmacological properties. 4-Substituted 1,4dihydropyridines (1,4-DHPs) are well known as $\mathrm{Ca}^{2+}$ channel blockers and emerged as one of the most important classes of drugs for the treatment of cardiovascular diseases, including hypertension. $^{3}$ 1,4-Dihydropyridines possess a variety of biological activities, such as, antiatherosclerotic, bronchodilator, vasodilator, hepatoprotective, antidiabetic, geroprotective, and antitumor agent. ${ }^{4 \mathrm{a}-\mathrm{d}}$ Recent literature survey revealed that 1,4-DHPs exhibit several medicinal 
applications which include neuroprotectant ${ }^{5 \mathrm{a}}$ and platelet anti-aggregatory activity, ${ }^{5 \mathrm{~b}}$ in addition cerebral antiischemic activity in the treatment of Alzheimer's disease ${ }^{5 \mathrm{c}}$ and as chemo sensitizer in tumor therapy. ${ }^{5 \mathrm{~d}}$ These examples clearly exeplicite the remarkable potential of novel DHP derivatives as a source of valuable drug candidates.

On the other hand, because of the pharmacological uses of polyhydroquinoline derivatives, their synthesis have been extensively investigated in the presence of organic solvents and catalysts. ${ }^{6-11}$ However, many of these methods suffers disadvantages such as harsh reaction conditions, long reaction times, the use of a large quantity of volatile organic solvents and generally leading to low yields. Recently, the synthesis of polyhydroquinoline derivatives have been carried out using TMSCl, ${ }^{12}$ ionic liquids, ${ }^{13 \mathrm{a}-\mathrm{b}}$ polymers ${ }^{1 \mathrm{a}-\mathrm{b}}$ and $\mathrm{Yb}(\mathrm{OTf})_{3},{ }^{15}$ and promotion of microwave, ${ }^{16} \mathrm{CAN},{ }^{17}$ solar thermal energy, ${ }^{18}$ potassium dodecatungsto cobaltate trihydrate, ${ }^{19}$ Baker's yeast, ${ }^{20}$ organocatalyst,${ }^{21} \mathrm{~K}_{7}\left[\mathrm{PW}_{11} \mathrm{CoO}_{40}\right] .{ }^{22}$ To the best our knowledge, the synthesis of polyhydroquinoline derivatives without catalyst in aqueous medium has not been reported so far. Consequently, there is a scope for further renovation of such synthetic methods, which avoids both an organic solvent and catalyst.

In this communication, we wish to report a simple, environmentally benign and efficient method for the one-pot synthesis of polyhydroquinoline derivatives from the reactions of aldehydes, dimedone, ethyl acetoacetate and ammonium acetate in refluxing water (Scheme1).

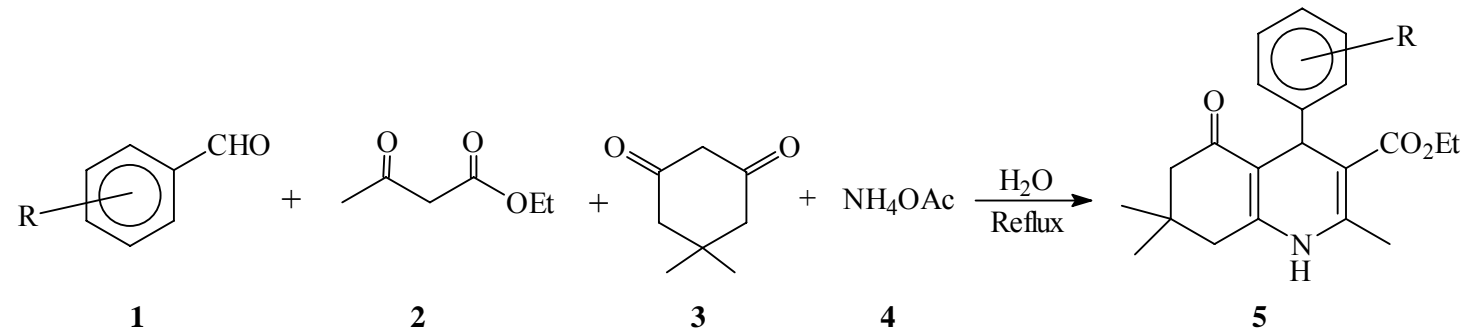

\section{Scheme 1}

The results are presented in Table 1 . When attempts were made to carry out the model reaction of benzaldehyde, dimedone, ethyl acetoacetate and ammonium acetate at room temperature in water, formation of solid product (5a) was observed, but in low yield (20\%) after $24 \mathrm{~h}$. The TLC of reaction mixture indicated the presence of starting materials. However, under reflux conditions the yield of product (5a) was improved significantly (96\%). This clearly demonstrates that temperature has important effect on the reaction and the best results were obtained at reflux temperature in water. It is also important to note that the reaction proceeded smoothly at room temperature in water and products were obtained in excellent yields. Thus, we have selected the optimized reaction conditions to examine the generality of this reaction. Various aromatic, and aliphatic aldehydes underwent smooth reaction with dimedone, ethyl acetoacetate and ammonium acetate to furnish excellent yields of products. The effect of electron-donating or withdrawing substituents on aromatic ring of aldehydes did not show any effect on rate of reaction and yield of the product. 
Table 1. Synthesis of polyhydroquinoline derivatives with various aldehydes in water under reflux conditions

\begin{tabular}{|c|c|c|c|c|c|}
\hline Entry & Aldehyde & Product & $\begin{array}{l}\text { Time } \\
\text { (h) }\end{array}$ & \begin{tabular}{|c|} 
Yielda,b \\
$(\%)$
\end{tabular} & $\mathrm{Mp}\left({ }^{\circ} \mathrm{C}\right)$ \\
\hline 1 & & & 8.00 & 96 & $228-229$ \\
\hline 2 & & & 4.50 & 98 & $260-262$ \\
\hline 3 & & & 5.00 & 99 & $245-246$ \\
\hline 4 & & & 4.50 & 96 & $265-266$ \\
\hline 5 & & & 5.50 & 95 & $182-184$ \\
\hline 6 & & & 5.00 & 97 & $241-242$ \\
\hline 7 & & & 6.00 & 97 & $210-211$ \\
\hline
\end{tabular}


Table 1. Continued

\begin{tabular}{|c|c|c|c|c|c|}
\hline Entry & Aldehyde & Product & $\begin{array}{l}\text { Time } \\
\text { (h) }\end{array}$ & $\begin{array}{l}\text { Yield,b }^{\mathrm{a}, \mathrm{b}} \\
(\%)\end{array}$ & $\mathrm{Mp}\left({ }^{\circ} \mathrm{C}\right)$ \\
\hline 8 & & & 3.75 & 98 & $108-110$ \\
\hline 9 & & & 6.25 & 97 & $288-290$ \\
\hline 10 & & & 2.25 & 90 & $200-202$ \\
\hline 11 & & & 4.50 & 95 & 216-218 \\
\hline 12 & & & 4.00 & 93 & $208-209$ \\
\hline
\end{tabular}


In conclusion, we have developed green protocol for four component cyclization reaction of aldehydes, dimedone, ethyl acetoacetate and ammonium acetate in water. This green procedure is environmentally benign involving water as green solvent and products are obtained by simple filtration.

\section{Experimental Section}

\section{A typical experimental procedure}

A mixture of 4-chlorobenzaldehyde $(2 \mathrm{mmol})$, dimedone $(2 \mathrm{mmol})$, ethyl acetoacetate $(2.5 \mathrm{mmol})$ and ammonium acetate $(3 \mathrm{mmol})$ in water $(6 \mathrm{ml})$ was refluxed for $5.0 \mathrm{~h}$. After completion of the reaction (TLC), the mixture was cooled and poured in crushed ice. The solid product was filtered to get almost pure product, which was crystallized from ethanol.

Ethyl-1,4,7,8-tetrahydro-2,7,7-4-(phenyl)-5-(6H)-oxoquinolin-3-carboxylate (5a). Solid; IR (KBr): 3292, 3210, 3074, 1699, 1602, 1067, 690; ${ }^{1} \mathrm{H}$ NMR $\left(\mathrm{CDCl}_{3}, 300 \mathrm{MHz}\right): \delta=0.90$ (s, 3H, $\left.\mathrm{C}-\mathrm{CH}_{3}\right), 1.01\left(\mathrm{~s}, 3 \mathrm{H}, \mathrm{C}-\mathrm{CH}_{3}\right), 1.20\left(\mathrm{t}, J=7.00 \mathrm{~Hz}, 3 \mathrm{H}, \mathrm{CH}_{3}-\mathrm{CH}_{2} \mathrm{O}\right), 2.03-206\left(\mathrm{~m}, 4 \mathrm{H}, 2 \mathrm{x} \mathrm{CH}_{2}\right)$, $2.38\left(\mathrm{~s}, 3 \mathrm{H},=\mathrm{C}-\mathrm{CH}_{3}\right), 4.01$ (q, $\left.J=6.8 \mathrm{~Hz}, 2 \mathrm{H}, \mathrm{OCH}_{2} \mathrm{CH}_{3}\right), 4.96(\mathrm{~s}, 1 \mathrm{H}, \mathrm{Ar}-\mathrm{CH}), 6.39$ (s, 5H, $\mathrm{NH}), 7.02-7.31(\mathrm{~m}, 5 \mathrm{H}, \mathrm{Ar}-\mathrm{H})$; MS: $m / z=340(\mathrm{M}+\mathrm{H})^{+}$.

Ethyl-1,4,7,8-tetrahydro-2,7,7-4-(4-methoxyphenyl)-5-(6H)-oxoquinolin-3-carboxylate (5b). Solid; IR (KBr): 3287, 3201, 3080, 1705, 1610, 1213, $836 \mathrm{~cm}^{-1} ;{ }^{1} \mathrm{H} \mathrm{NMR}\left(\mathrm{CDCl}_{3}, 300 \mathrm{MHz}\right): \delta$ $=0.91\left(\mathrm{~s}, 3 \mathrm{H}, \mathrm{C}-\mathrm{CH}_{3}\right), 1.04\left(\mathrm{~s}, 3 \mathrm{H}, \mathrm{C}-\mathrm{CH}_{3}\right), 1.20\left(\mathrm{t}, \mathrm{J}=6.8 \mathrm{~Hz}, 3 \mathrm{H}, \mathrm{CH}_{3}-\mathrm{CH}_{2} \mathrm{O}\right), 2.09-2.30(\mathrm{~m}$, $\left.4 \mathrm{H}, 2 \times \mathrm{CH}_{2}\right), 2.33\left(\mathrm{~s}, 3 \mathrm{H},=\mathrm{C}-\mathrm{CH}_{3}\right), 3.70\left(\mathrm{~s}, 3 \mathrm{H}, \mathrm{OCH}_{3}\right), 4.03\left(\mathrm{q}, J=6.9 \mathrm{~Hz}, \mathrm{OCH}_{2} \mathrm{CH}_{3}\right), 4.96$ (s, 1H, Ar-C-H), 6.39 (s, 1H, NH), 6.69 (d, J = 8.4, 2H, 2 x Ar-H), 7.17 (d, J = 8.4Hz, $2 \mathrm{H}, 2$ x ArH); MS: $m / z=370(\mathrm{M}+\mathrm{H})^{+}$.

Ethyl-1,4,7,8-tetrahydro-2,7,7-4-(4-chlorophenyl)-5-(6H)-oxoquinolin-3-carboxylate (5c). Solid; IR (KBr): 3290, 3205, 3074, 1705, 1608, 1073, $836 \mathrm{~cm}^{-1} ;{ }^{1} \mathrm{H}$ NMR $\left(\mathrm{CDCl}_{3}, 300 \mathrm{MHz}\right): \delta$ $=0.91\left(\mathrm{~s}, 3 \mathrm{H}, \mathrm{C}-\mathrm{CH}_{3}\right), 1.03\left(\mathrm{~s}, 3 \mathrm{H}, \mathrm{C}-\mathrm{CH}_{3}\right), 1.24\left(\mathrm{t}, J=6.8 \mathrm{~Hz}, 3 \mathrm{H}, \mathrm{CH}_{3}-\mathrm{CH}_{2} \mathrm{O}\right), 2.08-2.28(\mathrm{~m}$, $\left.4 \mathrm{H}, 2 \times \mathrm{CH}_{2}\right), 2.35\left(\mathrm{~s}, 3 \mathrm{H},=\mathrm{C}-\mathrm{CH}_{3}\right), 4.08$ (q, $\left.J=6.7 \mathrm{~Hz}, 2 \mathrm{H}, \mathrm{OCH}_{2} \mathrm{CH}_{3}\right), 4.97(\mathrm{~s}, 1 \mathrm{H}, \mathrm{Ar}-\mathrm{CH})$, $6.40(\mathrm{~s}, 1 \mathrm{H}, \mathrm{NH}), 7.01$ (d, $J=8.2 \mathrm{~Hz}, 2 \mathrm{H}, 2 \times \mathrm{Ar}-\mathrm{H}), 7.31$ (d, $J=8.2 \mathrm{~Hz}, 2 \mathrm{H}, 2 \times \mathrm{Ar}-\mathrm{H})$; MS: $\mathrm{m} / \mathrm{z}$ $=374(\mathrm{M}+\mathrm{H})^{+}$. .

Ethyl-1,4,7,8-tetrahydro-2,7,7-4-(4-methylphenyl)-5-(6H)-oxoquinolin-3-carboxylate (5d). Solid; IR (KBr): 3280, 3195, 3074, 1699, 1602, 1213, $1061 \mathrm{~cm}^{-1} ;{ }^{1} \mathrm{H}$ NMR $\left(\mathrm{CDCl}_{3}, 300 \mathrm{MHz}\right): \delta$ $=0.90\left(\mathrm{~s}, 3 \mathrm{H}, \mathrm{C}-\mathrm{CH}_{3}\right), 1.05\left(\mathrm{~s}, 3 \mathrm{H}, \mathrm{C}-\mathrm{CH}_{3}\right), 1.21\left(\mathrm{t}, J=7.00 \mathrm{~Hz}, 3 \mathrm{H}, \mathrm{CH}_{3}-\mathrm{CH}_{2} \mathrm{O}\right), 2.10(\mathrm{~s}, 3 \mathrm{H}$, $\left.=\mathrm{C}-\mathrm{CH}_{3}\right), 2.20-2.40\left(\mathrm{~m}, 4 \mathrm{H}, 2 \times \mathrm{CH}_{2}\right), 2.38\left(\mathrm{~s}, 3 \mathrm{H}, \mathrm{Ar}-\mathrm{CH}_{3}\right), 4.04(\mathrm{q}, 2 \mathrm{H}, J=7.00 \mathrm{~Hz}$, $\left.\mathrm{OCH}_{2} \mathrm{CH}_{3}\right), 4.97$ (s, 1H, Ar-CH), $6.40(\mathrm{~s}, 1 \mathrm{H}, \mathrm{NH}), 6.98(\mathrm{~d}, J=8.2 \mathrm{~Hz}, 2 \mathrm{H}, \mathrm{Ar}-\mathrm{H}), 7.01(\mathrm{~d}, J=$ $8.2 \mathrm{~Hz}, 2 \mathrm{H}, \mathrm{ArH})$; MS: $m / z=354(\mathrm{M}+\mathrm{H})^{+}$. .

Ethyl-1,4,7,8-tetrahydro-2,7,7-4-(3-nitrophenyl)-5-(6H)-oxoquinolin-3-carboxylate (5e). Solid; IR (KBr): 3299, 2958, 1687, 1610, 1164, $757 \mathrm{~cm}^{-1} ;{ }^{1} \mathrm{H}$ NMR $\left(\mathrm{CDCl}_{3}, 300 \mathrm{MHz}\right): \delta=0.92$ (s, 3H, C-CH3), $1.05\left(\mathrm{~s}, 3 \mathrm{H}, \mathrm{C}-\mathrm{CH}_{3}\right), 1.21\left(\mathrm{t}, \mathrm{J}=7.2 \mathrm{~Hz}, 3 \mathrm{H}, \mathrm{CH}_{3}-\mathrm{CH}_{2} \mathrm{O}\right), 2.10-2.31$ (m, 4H, 2 
x $\mathrm{CH}_{2}$ ), 2.36 (s, 3H, =C-CH $), 4.06\left(\mathrm{q}, J=6.8 \mathrm{~Hz}, 2 \mathrm{H}, \mathrm{OCH}_{2}\right), 4.95(\mathrm{~s}, 1 \mathrm{H}, \mathrm{Ar}-\mathrm{CH}), 6.36(\mathrm{~s}, 1 \mathrm{H}$, $\mathrm{NH}), 6.70(\mathrm{~d}, J=8.4 \mathrm{~Hz}, 1 \mathrm{H}, \mathrm{Ar}-\mathrm{H}), 7.02-7.15(\mathrm{~m}, 2 \mathrm{H}, 2 \mathrm{x} \mathrm{Ar}-\mathrm{H}), 7.38$ (d, $J=8.4 \mathrm{~Hz}, 1 \mathrm{H}, \mathrm{Ar}-$ $\mathrm{H})$; $\mathrm{MS}: m / z=385(\mathrm{M}+\mathrm{H})^{+}$. .

Ethyl-1,4,7,8-tetrahydro-2,7,7-4-(4-nitrophenyl)-5-(6H)-oxoquinolin-3-carboxylate (5f). Solid; IR (KBr): 3294, 3209, 3080, 1709, 1612, 1173, $840 \mathrm{~cm}^{-1} ;{ }^{1} \mathrm{H}$ NMR $\left(\mathrm{CDCl}_{3}, 300 \mathrm{MHz}\right): \delta$ $=0.93\left(\mathrm{~s}, 3 \mathrm{H}, \mathrm{C}-\mathrm{CH}_{3}\right), 1.06\left(\mathrm{~s}, 3 \mathrm{H}, \mathrm{C}-\mathrm{CH}_{3}\right), 1.26-1.28\left(\mathrm{t}, J=7.00 \mathrm{~Hz}, 3 \mathrm{H}, \mathrm{CH}_{3}-\mathrm{CH}_{2} \mathrm{O}\right), 2.09-$ $2.30\left(\mathrm{~m}, 4 \mathrm{H}, 2 \times \mathrm{CH}_{2}\right), 2.35\left(\mathrm{~s}, 3 \mathrm{H},=\mathrm{C}-\mathrm{CH}_{3}\right), 4.18\left(\mathrm{q}, J=6.7 \mathrm{~Hz}, 2 \mathrm{H}, \mathrm{OCH}_{2} \mathrm{CH}_{3}\right), 4.97(\mathrm{~s}, 1 \mathrm{H}$, Ar-CH), 6.36 (s, 1H, NH), 7.20 (d, $J=8.4 \mathrm{~Hz}, 2 \mathrm{H}, \mathrm{ArH}), 7.53$ (d, $J=8.4 \mathrm{~Hz}, 2 \mathrm{H}, \mathrm{ArH}) ; \mathrm{MS}: \mathrm{m} / \mathrm{z}$ $=385(\mathrm{M}+\mathrm{H})^{+}$.

Ethyl-1,4,7,8-tetrahydro-2,7,7-4-(3,4-methylenedioxyphenyl)-5-(6H)-oxoquinolin-3carboxylate (5g). Solid; IR (KBr): 3287, 3195, 3079, 1691, 1598, 1037, $862 \mathrm{~cm}^{-1}$; ${ }^{1} \mathrm{H}$ NMR $\left(\mathrm{CDCl}_{3}, 300 \mathrm{MHz}\right): \delta=0.91\left(\mathrm{~s}, 3 \mathrm{H}, \mathrm{C}-\mathrm{CH}_{3}\right), 1.03\left(\mathrm{~s}, 3 \mathrm{H}, \mathrm{C}-\mathrm{CH}_{3}\right), 1.20\left(\mathrm{t}, J=6.9 \mathrm{~Hz}, 3 \mathrm{H}, \mathrm{CH}_{3}-\right.$ $\left.\mathrm{CH}_{2} \mathrm{O}\right), 2.09-2.32\left(\mathrm{~m}, 4 \mathrm{H}, 4 \mathrm{H}, 2 \times \mathrm{CH}_{2}\right), 2.38\left(\mathrm{~s}, 3 \mathrm{H},=\mathrm{C}-\mathrm{CH}_{3}\right), 4.06(\mathrm{q}, J=6.9 \mathrm{~Hz}, 2 \mathrm{H}$, $\left.\mathrm{OCH}_{2} \mathrm{CH}_{3}\right), 4.92(\mathrm{~s}, 1 \mathrm{H}, \mathrm{Ar}-\mathrm{CH}), 6.03$ (s, 2H, $\left.\mathrm{OCH}_{2} \mathrm{O}\right), 6.33$ (s, 1H, NH), 7.65-7.69 (m, $2 \mathrm{H}, 2 \mathrm{x}$ $\mathrm{ArH}), 7.75(\mathrm{~d}, J=8.4 \mathrm{~Hz}, 1 \mathrm{H}, \mathrm{ArH}) ; \mathrm{MS}: m / z=384(\mathrm{M}+\mathrm{H})^{+}$. .

Ethyl-1,4,7,8-tetrahydro-2,7,7-4-(2-fluorophenyl)-5-(6H)-oxoquinolin-3-carboxylate (5h). Solid; IR (KBr): 3282, 3199, 3077, 1698, 1600, 1218, $755 \mathrm{~cm}^{-1} ;{ }^{1} \mathrm{H}$ NMR $\left(\mathrm{CDCl}_{3}, 300 \mathrm{MHz}\right): \delta$ $=0.91\left(\mathrm{~s}, 3 \mathrm{H}, \mathrm{C}-\mathrm{CH}_{3}\right), 1.05\left(\mathrm{~s}, 3 \mathrm{H}, \mathrm{C}-\mathrm{CH}_{3}\right), 1.23\left(\mathrm{t}, \mathrm{J}=6.9 \mathrm{~Hz}, 3 \mathrm{H}, \mathrm{CH}_{3}-\mathrm{CH}_{2} \mathrm{O}\right), 2.12(\mathrm{~s}, 3 \mathrm{H}$, $\left.=\mathrm{C}-\mathrm{CH}_{3}\right), 2.24\left(\mathrm{~m}, 4 \mathrm{H}, 2 \times \mathrm{CH}_{2}\right), 4.02\left(\mathrm{q}, J=6.9 \mathrm{~Hz}, 2 \mathrm{H}, \mathrm{OCH}_{2} \mathrm{CH}_{3}\right), 4.89(\mathrm{~s}, 1 \mathrm{H}, \mathrm{Ar}-\mathrm{CH}), 6.38$ (s, 1H, NH), $7.03-7.22(\mathrm{~m}, 4 \mathrm{H}, \mathrm{ArH})$; MS: $m / z=358(\mathrm{M}+\mathrm{H})^{+}$.

Ethyl-1,4,7,8-tetrahydro-2,7,7-4-(3,4-dimethoxyphenyl)-5-(6H)-oxoquinolin-3-carboxylate (5i). Solid; IR (KBr): 3280, 3201, 3074, 1693, 1614, 1018, $732 \mathrm{~cm}^{-1} ;{ }^{1} \mathrm{H} \mathrm{NMR}\left(\mathrm{CDCl}_{3}, 300\right.$ $\mathrm{MHz}): \delta=0.92\left(\mathrm{~s}, 3 \mathrm{H}, \mathrm{C}-\mathrm{CH}_{3}\right), 1.38\left(\mathrm{~s}, 3 \mathrm{H}, \mathrm{C}-\mathrm{CH}_{3}\right), 1.24\left(\mathrm{t}, J=7.00 \mathrm{~Hz}, 3 \mathrm{H}, \mathrm{CH}_{3}-\mathrm{CH}_{2} \mathrm{O}\right), 2.10-$ $2.30\left(\mathrm{~m}, 4 \mathrm{H}, 2 \times \mathrm{CH}_{2}\right), 2.35\left(\mathrm{~s}, 3 \mathrm{H},=\mathrm{C}-\mathrm{CH}_{3}\right), 3.82\left(\mathrm{~s}, 3 \mathrm{H}, \mathrm{OCH}_{3}\right), 3.86\left(\mathrm{~s}, 3 \mathrm{H}, \mathrm{OCH}_{3}\right), 4.10(\mathrm{q}, \mathrm{J}$ $\left.=8.4 \mathrm{~Hz}, 2 \mathrm{H}, \mathrm{OCH}_{2}\right), 5.00(\mathrm{~s}, 1 \mathrm{H}, \mathrm{Ar}-\mathrm{CH}), 6.36(\mathrm{~s}, 1 \mathrm{H}, \mathrm{NH}), 6.38(\mathrm{~d}, J=8.3 \mathrm{~Hz}, 1 \mathrm{H}), 6.76(\mathrm{~d}, J=$ $8.3 \mathrm{~Hz}, 1 \mathrm{H}), 6.92(\mathrm{~s}, 1 \mathrm{H}, \mathrm{Ar}-\mathrm{H})$; MS: $\mathrm{m} / \mathrm{z}=400(\mathrm{M}+\mathrm{H})^{+}$.

Ethyl-1,4,7,8-tetrahydro-2,7,7-4-(3-hydroxy,4-methoxyphenyl)-5-(6H)-oxoquinolin-3carboxylate (5j). Solid; IR (KBr): 3392, 3291, 3081, 1698, 1613, 1020, $735 \mathrm{~cm}^{-1}$; ${ }^{1} \mathrm{H}$ NMR $\left(\mathrm{CDCl}_{3}, 300 \mathrm{MHz}\right): \delta=0.92\left(\mathrm{~s}, 3 \mathrm{H}, \mathrm{C}-\mathrm{CH}_{3}\right), 1.02\left(\mathrm{~s}, 3 \mathrm{H}, \mathrm{C}-\mathrm{CH}_{3}\right), 1.21\left(\mathrm{t}, J=6.8 \mathrm{~Hz}, 3 \mathrm{H}, \mathrm{CH}_{3^{-}}\right.$ $\left.\mathrm{CH}_{2}\right), 2.12-2.37\left(\mathrm{~m}, 4 \mathrm{H}, 2 \times \mathrm{CH}_{2}\right), 2.39\left(\mathrm{~s}, 3 \mathrm{H},=\mathrm{C}_{-} \mathrm{CH}_{3}\right), 3.68\left(\mathrm{~s}, 3 \mathrm{H}, \mathrm{OCH}_{3}\right), 4.04(\mathrm{q}, J=6.8$ $\left.\mathrm{Hz}, \mathrm{OCH}_{2} \mathrm{CH}_{3}\right), 4.98(\mathrm{~s}, 1 \mathrm{H}, \mathrm{Ar}-\mathrm{CH}), 5.37(\mathrm{~s}, 1 \mathrm{H}, \mathrm{OH}), 6.40(\mathrm{~s}, 1 \mathrm{H}, \mathrm{NH}), 6.72-6.70$ (m, $2 \mathrm{H}, 2 \mathrm{x}$ Ar-H), $7.02(\mathrm{~d}, J=8.2 \mathrm{~Hz}, 1 \mathrm{H}, \mathrm{ArH})$; MS: $m / z=386(\mathrm{M}+\mathrm{H})^{+}$.

Ethyl-1,4,7,8-tetrahydro-2,7,7-4-(2,4-dichlorophenyl)-5-(6H)-oxoquinolin-3-carboxylate

(5k). Solid; IR (KBr): 3291, 3203, 3088, 1702, 1605, 1100, $859 \mathrm{~cm}^{-1} ;{ }^{1} \mathrm{H} \mathrm{NMR}\left(\mathrm{CDCl}_{3}, 300\right.$ $\mathrm{MHz}): \delta=0.93\left(\mathrm{~s}, 3 \mathrm{H}, \mathrm{C}-\mathrm{CH}_{3}\right), 1.04\left(\mathrm{~s}, 3 \mathrm{H}, \mathrm{C}-\mathrm{CH}_{3}\right), 1.23\left(\mathrm{t}, \mathrm{J}=6.8 \mathrm{~Hz}, 3 \mathrm{H}, \mathrm{CH}_{3}-\mathrm{CH}_{2} \mathrm{O}\right), 2.36-$ $2.60\left(\mathrm{~m}, 4 \mathrm{H}, 2 \times \mathrm{CH}_{2}\right), 2.93\left(\mathrm{~s}, 3 \mathrm{H},=\mathrm{C}-\mathrm{CH}_{3}\right), 4.09\left(\mathrm{q}, J=6.8 \mathrm{~Hz}, 2 \mathrm{H}, \mathrm{OCH}_{2} \mathrm{CH}_{3}\right), 4.83(\mathrm{~s}, 1 \mathrm{H}$, Ar-CH), $6.36(\mathrm{~s}, 1 \mathrm{H}, \mathrm{NH}), 7.69-7.73(\mathrm{~m}, 2 \mathrm{H}, 2 \mathrm{x} \mathrm{ArH}), 7.82(\mathrm{~d}, J=8.4 \mathrm{~Hz}, 1 \mathrm{H}, \mathrm{ArH}) ; \mathrm{MS}: \mathrm{m} / \mathrm{z}=$ $409(\mathrm{M}+\mathrm{H})^{+}$.

Ethyl-1,4,7,8-tetrahydro-2,7,7-4-(2-chlorophenyl)-5-(6H)-oxoquinolin-3-carboxylate (5l). Solid; IR (KBr): 3296, 3207, 3092, 1707, 1610, 1156, $830 \mathrm{~cm}^{-1} ;{ }^{1} \mathrm{H}$ NMR $\left(\mathrm{CDCl}_{3}, 300 \mathrm{MHz}\right): \delta$ 
$=0.91\left(\mathrm{~s}, 3 \mathrm{H}, \mathrm{C}-\mathrm{CH}_{3}\right), 1.02\left(\mathrm{~s}, 3 \mathrm{H}, \mathrm{C}-\mathrm{CH}_{3}\right), 1.20-1.26\left(\mathrm{t}, \mathrm{J}=7.00 \mathrm{~Hz}, 3 \mathrm{H}, \mathrm{CH}_{3}-\mathrm{CH}_{2} \mathrm{O}\right), 2.09-$ $2.30\left(\mathrm{~m}, 4 \mathrm{H}, 2 \times \mathrm{CH}_{2}\right), 2.33\left(\mathrm{~s}, 3 \mathrm{H},=\mathrm{C}-\mathrm{CH}_{3}\right), 4.01\left(\mathrm{q}, J=6.4 \mathrm{~Hz}, 2 \mathrm{H}, \mathrm{OCH}_{2} \mathrm{CH}_{3}\right), 4.88(\mathrm{~s}, 1 \mathrm{H}$, Ar-CH), 6.35 (s, 1H, NH), $7.01-7.20(\mathrm{~m}, 4 \mathrm{H}, \mathrm{ArH}) \mathrm{MS}: \mathrm{m} / \mathrm{z}=374(\mathrm{M}+\mathrm{H})^{+}$.

Ethyl-1,4,7,8-tetrahydro-2,7,7-4-(hexanyl)-5-(6H)-oxoquinolin-3-carboxylate (5m). Solid; IR (Neat): 3289, 3195, 3080, 1696, 1598, 1037, $922 \mathrm{~cm}^{-1} ;{ }^{1} \mathrm{H} \mathrm{NMR}\left(\mathrm{CDCl}_{3}, 300 \mathrm{MHz}\right): \delta=0.89$ (s, $\left.3 \mathrm{H}, \mathrm{C}-\mathrm{CH}_{3}\right), 1.02\left(\mathrm{~s}, 3 \mathrm{H}, \mathrm{C}-\mathrm{CH}_{3}\right), 1.19\left(\mathrm{t}, 3 \mathrm{H}, J=7.5 \mathrm{~Hz}, \mathrm{CH}_{3} \mathrm{CH}_{2} \mathrm{O}\right), 1.21-1.5(\mathrm{~m}, 11 \mathrm{H}$, $\mathrm{CH}_{2} \mathrm{CH}_{2} \mathrm{CH}_{2} \mathrm{CH}_{2} \mathrm{CH}_{3}$ ), $2.11\left(\mathrm{~s}, 3 \mathrm{H},=\mathrm{C}-\mathrm{CH}_{3}\right), 2.23-2.40\left(\mathrm{~m}, 2 \mathrm{H}, 2 \times \mathrm{CH}_{2}\right), 4.01$ (q, $2 \mathrm{H}, J=7.5$ $\left.\mathrm{Hz}, \mathrm{OCH}_{2} \mathrm{CH}_{3}\right), 4.21(\mathrm{~s}, 1 \mathrm{H}, \mathrm{Ar}-\mathrm{CH}), 6.33(\mathrm{~s}, 1 \mathrm{H}, \mathrm{NH}) ; \mathrm{MS}: m / z=334(\mathrm{M}+\mathrm{H})^{+}$.

\section{Acknowledgements}

We are thankful to Council of Scientific and Industrial Research (CSIR), New Delhi (Project no. 1/2023/05/EMR-II) for the financial assistance and PEM thanks UGC, New Delhi for teacher fellowship under faculty improvement program.

\section{References}

1. Hudlicky, T. Chem. Rev. 1996, 96, 3.

2. (a) Naik, S.; Bhattacharjya, G.; Talukdar, B.; Patel. B. K. Eur. J. Org. Chem. 2004, 1245. (b) Naik, S.; Bhattacharjya, G.; Kavala, V. R.; Patel B. K. ARKIVOC 2004, 55.

3. (a) Bossert, F.; Meyer, H.; Wehinger, E. Angew. Chem., Int. Ed. 1981, 20, 762. (b) Nakayama, H.; Kasoaka, Y. Heterocycles 1996, 42, 901.

4. (a) Godfraid, T.; Miller, R.; Wibo, M. Pharmocol. Rev. 1986, 38, 321. (b) Sausins, A.; Duburs, G. Heterocycles 1988, 27, 269. (c) Mager, P. P.; Coburn, R. A.; Solo, A. J.; Triggle, D. J.; Rothe, H. Drug Design Discovery 1992, 8, 273. (d) Mannhold, R.; Jablonka, B.; Voigdt, W.; Schoenafinger, K.; Schravan, K. Eur. J. Med. Chem. 1992, 27, 229.

5. (a) Klusa, V. Drugs Fut. 1995, 20, 135. (b) Bretzel, R. G.; Bollen, C. C.; Maeser, E.; Federlin, K. F. Am. J. Kidney. Dis. 1993, 21, 53. (c) Bretzel, R. G.; Bollen, C. C.; Maeser, E.; Federlin, K. F. Drugs Fut. 1992, 17, 465. (d) Boer, R.; Gekeler, V. Drugs Fut. 1995, $20,499$.

6. Hantzsch, A. Ann. Chem. 1882, I, 215.

7. Sainani, J. B.; Shah, A. C.; Arya, V. P. Indian J. Chem, Sect B 1994, 33, 526.

8. Ahluwalia, V. K.; Goyal, B.; Das, U. J. Chem. Res., Synop. 1997, 266.

9. Margarita, S.; Estael, O.; Yamila, V.; Beatriz, P.; Lourdes, M.; Nazario, M.; Margarita. Q.; Carlos, S.; Jose, L. S.; Hector, N.; Norbert, B.; Oswald, M. P. Tetrahedron 1999, 55, 875.

10. Ahluwalia, V. K.; Goyal, B. Das, U. J. Chem. Res., Miniprint. 1997, 7, 1501.

11. Ahluwalia, V. K.; Goyal, B. Indian J. Chem, Sect. B 1996, 35, 1021.

12. Sabitha, G.; Reddy, G. S. K. K.; Reddy, C. S.; Yadav, J. S. Tetrahedron Lett. 2003, 44, 4129. 
13. (a) Ji, S. J.; Jiang, Z. Q.; Lu, J.; Loa, T. P. Synlett 2004, 831. (b) Sridhar, R.; Perumal, P. T. Tetrahedron 2005, 61, 2465.

14. (a) Breitenbucher, J. G.; Figliozzi, G. Tetrahedron Lett. 2000, 41, 4311. (b) Dondoni, A.; Massi, A.; Minghini, E.; Bertolasi, V. Tetrahedron 2004, 60, 2311.

15. Wang, L. M.; Sheng, J.; Zhang, L.; Han, J. W.; Fan, Z. Y.; Tian, H.; Qian, C. T.Tetrahedron 2005, 61, 1539.

16. Tu, S.-J.; Zhou, J.-F.; Deng, X.; Cai, P.-J.; Wang, H.; Feng, J.-C. Chin. J. Org. Chem. 2001, $21,313$.

17. Reddy, C. S.; Raghu, M. Chinese Chem. Lett. 2008 (In Press).

18. Mekheimer, R. A.; Hameed, A. A.; Sadek, K. U. Green Chem. 2008, 10(5), 592.

19. Nagarapu, L.; Apuri, S.; Gaddam, S.; Bantu, R.; Mahankhali, V. C.; Kantevari, S. Letters In Org. Chem. 2008, 5(1), 60.

20. Kumar, A.; Maurya, R. A. Tetrahedron Lett. 2007, 48(22), 3887.

21. Kumar, A.; Maurya, R. A. Tetrahedron 2008, 63(9), 1946.

22. Heravi, M. M.; Bakhtiari, K.; Javadi, N. M.; Bamoharram, F. F.; Saeedi, M.; Oskooie, H. A. J. Mol. Cat. A: Chem. 2007, 264(1-2), 50. 